\title{
Framework for personalized prediction of treatment response in relapsing remitting multiple sclerosis
}

\author{
E. Stühler ${ }^{1}$, S. Braune ${ }^{2 *}$, F. Lionetto ${ }^{1}$, Y. Heer ${ }^{1}$, E. Jules ${ }^{1}$, C. Westermann ${ }^{1}$, A. Bergmann ${ }^{2}$, P. van Hövell ${ }^{1}$ and \\ NeuroTransData Study Group
}

\begin{abstract}
Background: Personalized healthcare promises to successfully advance the treatment of heterogeneous neurological disorders such as relapsing remitting multiple sclerosis by addressing the caveats of traditional healthcare. This study presents a framework for personalized prediction of treatment response based on real-world data from the NeuroTransData network.

Methods: A framework for personalized prediction of response to various treatments currently available for relapsing remitting multiple sclerosis patients was proposed. Two indicators of therapy effectiveness were used: number of relapses, and confirmed disability progression. The following steps were performed: (1) Data preprocessing and selection of predictors according to quality and inclusion criteria; (2) Implementation of hierarchical Bayesian generalized linear models for estimating treatment response; (3) Validation of the resulting predictive models based on several performance measures and routines, together with additional analyses that focus on evaluating the usability in clinical practice, such as comparing predicted treatment response with the empirically observed course of multiple sclerosis for different adherence profiles.
\end{abstract}

Results: The results revealed that the predictive models provide robust and accurate predictions and generalize to new patients and clinical sites. Three different out-of-sample validation schemes (10-fold cross-validation, leave-one-site-out cross-validation, and excluding a test set) were employed to assess generalizability based on three different statistical performance measures (mean squared error, Harrell's concordance statistic, and negative log-likelihood). Sensitivity to different choices of the priors, to the characteristics of the underlying patient population, and to the sample size, was assessed. Finally, it was shown that model predictions are clinically meaningful.

Conclusions: Applying personalized predictive models in relapsing remitting multiple sclerosis patients is still new territory that is rapidly evolving and has many challenges. The proposed framework addresses the following challenges: robustness and accuracy of the predictions, generalizability to new patients and clinical sites and comparability of the predicted effectiveness of different therapies. The methodological and clinical soundness of the results builds the basis for a future support of patients and doctors when the current treatment is not generating the desired effect and they are considering a therapy switch.

Keywords: Relapsing remitting multiple sclerosis, Personalized medicine, Clinical decision support, Personalized health record, Personalized predictive models, Bayesian generalized linear model

\footnotetext{
* Correspondence: sbraune@neurotransdata.com

${ }^{2}$ NeuroTransData, Neuburg an der Donau, Germany

Full list of author information is available at the end of the article
}

(c) The Author(s). 2020 Open Access This article is distributed under the terms of the Creative Commons Attribution 4.0 International License (http://creativecommons.org/licenses/by/4.0/), which permits unrestricted use, distribution, and reproduction in any medium, provided you give appropriate credit to the original author(s) and the source, provide a link to the Creative Commons license, and indicate if changes were made. The Creative Commons Public Domain Dedication waiver (http://creativecommons.org/publicdomain/zero/1.0/) applies to the data made available in this article, unless otherwise stated. 


\section{Background}

Knowledge about the effectiveness of available treatments is typically based on results from randomized controlled trials (RCTs). However, these results are derived in a controlled, constrained setting and do not necessarily reflect real-world patient populations and drug labels. In addition, results from RCTs are based on group-level differences, while treatment decisions require individual-level information to enable optimal treatment allocation for an individual patient. This can cause a 'trial-and-error paradigm of treatment allocation' [1], in which the individual patient undergoes several therapy switches until a suitable treatment is found. Real-world data are gaining increasing importance to fill this gap between RCTs and utilization of treatment options in daily practice. This is also why the European Medical Agency and the Food and Drug Administration in the USA are seeing the availability of real-world data as an emerging opportunity to improve treatment quality and allocation of resources [2] [3] [4] [5] [6]. In the field of multiple sclerosis (MS), several registries have captured qualified clinical data for more than 10 years, including MSBase (founded in 2004, multinational), OFSEP (2001, France), Swedish MS registry (2001, Sweden), MSDS3D (2010, Germany), and NeuroTransData (NTD; 2008, Germany).

MS is the most prevalent neurological auto-immune disorder of the central nervous system and affects patients in the most dynamic and productive time of their lives by causing severe physical disability and mental handicap, thus impairing abilities for social and professional participation over time in the majority of those affected. The treatment landscape of MS is continually changing: since 2000, more than 900 clinical studies have been listed in the clinical trials registry created by the National Institutes of Health alone. Following the first Interferon- $\beta 1 b$ injectable therapy in 1995, 14 different disease modifying therapies (DMTs) based on eight approved compounds became available in the EU in 2017 for the relapsing remitting form of multiple sclerosis (RRMS) [2].

Disease mechanisms and course of RRMS are heterogeneous and challenging to predict at a group level, and even more in an individual patient. This heterogeneity, the impact of clinical outcomes on quality of life and the large number of treatment options make a personalized approach for a tailored treatment of patients desirable.

This study is built on previous research on personalized medicine [5-10]. It contributes to an ongoing advancement on the personalization of RRMS treatment allocation by proposing a framework for comparing the effectiveness of DMTs at patient level. Two indicators of treatment effectiveness are taken into account: the number of ontherapy relapses experienced by the patient, and the occurrence of a confirmed disability progression (CDP) during the therapy. The statistical approach relies on hierarchical Bayesian generalized linear models (GLMs).
This study is based on the NTD MS registry, where physicians in Germany capture quality-proven real-world data in a large number of different clinical sites and for heterogeneous patients and disease histories. This provides the basis for the generalization of the predictive models to a wide range of patients and clinical sites that were not part of the model development. The methodological and clinical soundness of the results of this framework is thoroughly evaluated in addition by comparing the predicted treatment response with the clinically observed course of MS for different adherence profiles.

Applying personalized predictive models in RRMS patients is still a new territory that is rapidly evolving and has many challenges. The objective of this study is to address some of these challenges by providing robust and reliable predictions of treatment response based on realworld data. This builds the basis for a future support of patients and doctors when the ongoing treatment fails and a therapy switch is considered.

\section{Methods}

This study proposes a framework for personalized prediction of effectiveness of various therapies currently available for RRMS patients. The framework comprises the following steps: (i) data preprocessing, selection of predictors according to quality and inclusion criteria, and definition of two indicators of therapy effectiveness (Section Methods: Data); (ii) model development using hierarchical Bayesian GLMs for estimating therapy response according to both indicators of therapy effectiveness (Section Methods: Model development); (iii) model performance assessment based on state-of-the-art performance measures and procedures, together with additional analyses that focus on applicability in clinical practice, i.e. on generalizability to new data and on comparability of predictions for different treatments (Section Methods: Model performance assessment).

\section{Data}

This study employed clinical real-world data recorded in the NTD MS registry. NTD is a Germany-wide network of physicians in the fields of neurology and psychiatry that was founded in 2008. Currently, 153 neurologists in 78 offices work in NTD practices serving about 600,000 outpatients per year. Each practice is certified according to network-specific and ISO 9001 criteria. Compliance with these criteria is audited annually by an external certified audit organization. The NTD MS registry includes about 25,000 patients with MS, which represents about $15 \%$ of all MS patients in Germany. In this database, demographic and clinical parameters are captured in real time over an average of 3.7 visits and Expanded Disability Status Scale (EDSS) assessments per year per patient. Standardized clinical assessments of functional system scores and EDSS calculation are performed by 
certified raters (http://www.neurostatus.net). All personnel undergoes regular training to ensure quality of data in the database. This quality is monitored by the NTD data management team. Data input is checked for inconsistencies and errors by also using an error analysis program. Both automatic and manually executed queries are implemented to further ensure data quality, e.g. checks for inconsistencies and requests for missing information. All data are pseudonymized and pooled to form the NTD MS database. The codes uniquely identifying patients are managed by the Institute for medical information processing, Biometry and Epidemiology (Institut für medizinische Informationsverarbeitung, Biometrie und Epidemiologie (IBE)) at the Ludwig Maximilian University in Munich, Germany, acting as an external trust center. The data acquisition protocol described above was approved by the ethical committee of the Bavarian Medical Board (Bayerische Landesärztekammer; June 14, 2012) and re-approved by the ethical committee of the Medical Board North-Rhine (Ärztekammer Nordrhein; April 25, 2017).

For this study, data were extracted from the NTD MS database on July 1, 2018.

\section{Predictors}

The predictors that were used to model therapy effectiveness are listed in Table 1. An overview of their distribution and discretization is provided in Additional file 1: Table S1.3. All predictors were defined and selected based on prior scientific research and clinical expertise (SB, AB) [11].

\section{Data quality and inclusion criteria}

The data used for model development consist of therapy cycles, i.e. each observation corresponds to a therapy cycle. Several quality and inclusion criteria were applied for data preprocessing and patient population selection, respectively.

The selected observations fulfilled quality criteria related to validity, accuracy, completeness, consistency and uniformity of the information in the database, including: all predictors were available at the start of the index therapy, at least one relapse was documented before the start of the index therapy, patients were required to have at least one documented EDSS measurement before the start of the index therapy. Extreme therapy cycles with annualized relapse rate above 12 per year were excluded from the study.

The following inclusion criteria were applied: patients were required to be at least 18 years old, EDSS before the start of the index therapy was required to be less than or equal to six, index therapy was required to be one of the following: Dimethylfumarat (DMF), Fingolimod (FTY), Glatirameracetat (GA), Interferon-ß1 (IF), Natalizumab (NA) or Teriflunomide (TERI). Therapies that were prescribed within 6 months of MS diagnosis without a previous treatment failure were excluded as they do not represent therapy switches during the course of RRMS. If more than one therapy cycle was available for a single patient, one therapy was randomly selected, while the others were discarded. Therapy cycles corresponding to clinical sites with only one remaining patient were also excluded.

After the quality and inclusion criteria were applied, 90\% of the therapy cycles (3119) were used for model development and validation, and $10 \%$ of the therapy

Table 1 List of model predictors, along with code names for shorter reference across the study

\begin{tabular}{|c|c|}
\hline Code name & Description \\
\hline Age & Age at the start of the index therapy \\
\hline Gender & Gender \\
\hline EDSS & $\begin{array}{l}\text { EDSS (measured at most } 6 \text { months before or } 3 \text { months after the start of the therapy cycle, } \\
\text { and at least } 84 \text { days after a relapse) }\end{array}$ \\
\hline Index / Index therapy & DMT taken during the therapy cycle \\
\hline Current / Current therapy & DMT taken prior to the start of the therapy cycle \\
\hline Diagnosis distance & Time elapsed between MS diagnosis and start of index therapy \\
\hline Relapse distance & $\begin{array}{l}\text { Time elapsed between the last relapse preceding the start of the index therapy and the start } \\
\text { of the index therapy }\end{array}$ \\
\hline Relapses count & Number of relapses in the year prior to the start of the index therapy \\
\hline DMTs count & Number of DMTs taken prior to the start of the index therapy \\
\hline Second-line & Whether a second-line DMT has been taken before the start of the index therapy \\
\hline Current duration & Duration of the current therapy \\
\hline Index duration & Duration of the index therapy \\
\hline Clinical site & Clinical site where the course of MS is observed \\
\hline
\end{tabular}

EDSS expanded disability status scale, DMT disease modifying therapy, Second-line DMT to be employed by label of the European Medical Agency if previous DMT failed to achieve sufficient control of disease activity (of the DMTs considered in this work, this applies to Fingolimod and Natalizumab), MS multiple sclerosis. 
cycles (314) were used as test set for validation as described below in Section Methods: Model performance assessment: Model generalizability.

A detailed overview of the data selection process is shown in Additional file 1: Table S1.1. A comparison of the responses of interest and of the predictors before and after the quality and inclusion criteria were applied is presented in Additional file 1: Tables S1.2, S1.3 and Figure S1.3.

\section{Indicators of therapy effectiveness}

Number of relapses and confirmed disability progression (CDP) during the observation time of a therapy cycle are established clinical indicators for therapy effectiveness in RRMS [7, 8]. Both indicators were therefore used to measure the effectiveness of index therapies. Additionally, probabilities of being free of relapse and free of CDP, respectively, were derived for validation [9].

A CDP was defined as a worsening of at least 1.0 point when the previous EDSS is 5.5 or lower and 0.5 point otherwise; the worsening must be sustained for at least 3 months and must be confirmed by at least one other valid EDSS measurement. A more detailed definition of CDP is included in Additional file 2.

\section{Model development}

The number of on-therapy relapses was modelled as following a negative binomial distribution whose mean and shape parameters depend on individual patient characteristics and index therapy. The occurrence of a CDP was modelled as following a binomial distribution, where the probability of observing a CDP depends on individual patient characteristics and index therapy.

In both cases, a hierarchical Bayesian GLM was employed [12]. The correlation that typically arises between measurements coming from the same clinical site was addressed by modeling a random intercept. The duration of each observed therapy cycle was incorporated in the models as an offset term, since the number of relapses and the probability of observing a CDP is expected to be larger for longer exposure, i.e. observation time of index therapy. A detailed description of the models is presented in Additional file 3.

In this study, Bayesian estimation was used due to the advantages offered by the possibility to incorporate prior information, which also allows for regularization [13]. The specific values that were given to the parameters' priors are summarized in
Table 2. These priors are weakly informative, in line with the values proposed by [14]. The parameters are assumed to be independent.

Models were fitted with version 2.14.1 of the rstanarm package in $R$ [13]. This implementation uses the Hamiltonian Monte Carlo approach to draw samples from the parameters' posterior joint distribution. For each Markov chain started to this purpose, the convergence to the target distribution was assessed using the Gelman and Rubin potential scale reduction statistic $\hat{R}$ [13]. For each of the samples and for each of the six considered index therapies (Section Methods: Data: Data quality and inclusion criteria), the number of relapses or the occurrence of a CDP were predicted for each observation by disregarding clinicspecific random effects, i.e. by setting all random intercepts to zero (in rstanarm, this is done by setting the 're.form' argument of the posterior_predict function to ' $\sim 0$ '). A new patient will thereby have consistent predictions across different clinical sites. These predictions obtained from the posterior distribution were summarized by looking (i) at their average and at the fraction of those that predict an absence of relapse (relapse model), and (ii) at the fraction of those that predict an absence of CDP (CDP model).

\section{Model performance assessment}

In this section, the following content is presented: model calibration statistical measures of model performance model generalizability, comparison with nested models of lower complexity, sensitivity of the models to different choices of the priors, to the characteristics of the patient population, and to the sample size and comparison of treatment effectiveness predicted by the models.

\section{Model calibration}

The agreement between the predicted and observed outcomes was assessed by distributing the therapy cycles into several bins of predicted outcomes. The bin size was chosen such that there are 20 equally-populated bins in total, covering the full range of the predicted outcomes. For each bin, the mean predicted outcome was compared with the mean observed outcome. If the model is well-calibrated, the two quantities are expected to be close to each other. The agreement between predictions and observations was studied for all therapy cycles and also for each DMT separately. The adoption of equally-populated bins rather than equally-sized bins has the advantage that the statistical uncertainty due to the

Table 2 Default priors assigned to the relapse and CDP models' parameters

\begin{tabular}{lcccc}
\hline Model & Intercept & Fixed effects & Dispersion & Standard deviation of random intercepts \\
\hline Relapse & $\mathrm{N}(0,10)$ & $\mathrm{N}(0,2.5)$ & Half-Cauchy $(0,5)$ & Gamma $(1,1)$ \\
CDP & $\mathrm{N}(0,10)$ & $\mathrm{N}(0,2.5)$ & - & Gamma $(1,1)$ \\
\hline
\end{tabular}

CDP confirmed disability progression. 
population size of each bin is the same for all points in the calibration plot.

\section{Statistical measures of model performance}

Model performance was evaluated via mean squared error (MSE), negative log-likelihood and Harrell's concordance statistic (C-Index).

The C-Index was used to analyze the ability of the models to discriminate among different responses, in this case to discriminate between none and at least one relapse, and between the occurrence and absence of a $\mathrm{CDP}$, respectively. When comparing predicted and observed indicators of therapy effectiveness, therapy cycles with roughly the same duration were matched. This is achieved by allowing for up to 6 months difference if the smaller of the two durations is less than half a year, and up to 12 months difference otherwise [15].

The negative log-likelihood per patient was obtained following the approach in [12], page 169, and using the $\log$ lik function of the rstanarm package [13]. The negative log-likelihood for the full patient population was obtained by summing the negative log-likelihoods per patient.

Although the models allow to make predictions for the effectiveness of all six therapies included in this study (Section Methods: Data: Data quality and inclusion criteria), statistical measures were only evaluated where the associated indicator of therapy effectiveness could be observed, i.e. using the predictions for the observed index therapy.

\section{Model generalizability}

The generalizability of the models was assessed using three different out-of-sample validation schemes. The first validation scheme consisted in evaluating the model performance using a 10 -fold cross-validation. The second validation scheme used a leave-one-site-out crossvalidation with respect to the clinical site. Patients from the same clinical site were excluded from the sample that was used to fit the model and then used to test how well this model performs. The procedure was repeated for each clinical site. The third validation scheme evaluated the model performance on the test set.

Performance measures were calculated using out-ofsample predictions as well as in-sample predictions. Each in-sample prediction was obtained from one randomly selected training fold. Therefore, exactly one outof-sample and one in-sample prediction per therapy cycle were retained. Out-of-sample and in-sample performance measures were compared in order to identify overfitting. The robustness of the performance measures was assessed by repeating the 10 -fold cross-validation 40 times, which allowed to compute standard errors.

The modeling approach described in Section Methods: Model development leads to the generation of six predictions per patient, one for each of the six therapies under consideration. Only predictions for the observed index therapy were retained when analyzing generalizability.

\section{Comparison with nested models}

The models presented above allow to make comparable predictions for all six therapies included in this study for each patient and each indicator of therapy effectiveness. The impact of the patient characteristics and their interactions with the index therapy on the model predictions was evaluated by comparing the model of Section Methods: Model development with two models of lower complexity. These two models were nested in the predictive model.

The first nested model, referred to as non-personalized model, does not have a dependency on the patient characteristics (Table 3). This model returns a fixed ranking of the six therapies under consideration. The model is not personalized, since two patients with a therapy cycle of the same duration but different characteristics will obtain the same predicted response, and hence will have the same comparative therapy effectiveness profile.

The second nested model, referred to as prognostic model, has a dependency on the patient characteristics but not on their interactions with the index therapy (Table 3).

This model is an extension of the non-personalized model that additionally allows for personalization, that is, for patient characteristics to have an impact on the predicted response. However, it is important to note here that patient characteristics are not allowed to interact with the index therapy, i.e. there is no personalization in the obtained ranking of the six therapies under consideration.

The predictive model in this study differs from the prognostic model by the addition that individual patient characteristics were allowed to interact with the therapy, i.e. the therapy effectiveness and corresponding ranking were allowed to differ for different patients.

\section{Sensitivity analysis}

The predictions' robustness was tested with respect to different choices of the priors, to the characteristics of the underlying patient population, and to sample size. Methods and results are presented in detail in Additional file 5, Additional file 6 and Additional file 7.

\section{Comparison of predicted therapy response}

The modeling approach presented above leads to the generation of six predictions per patient (Section Methods: Model development), one for each of the six therapies under consideration. As both predictive models allow therapy effectiveness to differ for different patient characteristics, a personalized ranking of therapies was obtained for each patient. Note that this ranking only applies with respect to the chosen indicator of 
Table 3 Overview of the predictors used for predictive models and nested models

\begin{tabular}{|c|c|c|c|}
\hline & Non-personalized model & Prognostic model & Predictive model \\
\hline Clinical site & $x$ & $x$ & $x$ \\
\hline Index therapy & $x$ & $x$ & $x$ \\
\hline Index duration & $x$ & $x$ & $x$ \\
\hline Age & & $x$ & $x$ \\
\hline Gender & & $x$ & $x$ \\
\hline EDSS & & $x$ & $x$ \\
\hline Second-line & & $x$ & $x$ \\
\hline Current therapy & & $x$ & $x$ \\
\hline Current duration & & $x$ & $x$ \\
\hline Interaction (Current therapy, Current duration) & & $x$ & $x$ \\
\hline Diagnosis distance & & $x$ & $x$ \\
\hline Relapse distance & & $x$ & $x$ \\
\hline Relapses count & & $x$ & $x$ \\
\hline DMTs count & & $x$ & $x$ \\
\hline Interaction (Index therapy, Diagnosis distance) & & & $x$ \\
\hline Interaction (Index therapy, Gender) & & & $x$ \\
\hline Interaction (Index therapy, Relapses count) & & & $x$ \\
\hline Interaction (Index therapy, Second-line) & & & $x$ \\
\hline
\end{tabular}

EDSS expanded disability status scale, DMT disease modifying therapy, Second-line DMT to be employed by label of the European Medical Agency if previous DMT failed to achieve sufficient control of disease activity.

therapy effectiveness, i.e. in this case either the lowest predicted number of relapses or the lowest predicted probability of observing a $\mathrm{CDP}$, and does not represent an overall therapy recommendation which would account for multiple determinants. In order to evaluate the usability of the models in clinical practice, average observed treatment responses were compared between patients who received the highest ranked therapy (denoted as $D M T^{*}$ in the following) and those who did not.

To avoid potential confounders on responses, the distribution of each predictor was matched between patients who received $\mathrm{DMT}^{*}$ and those who did not with a propensity-score-based weighting as implemented by the twang package [16, 17]. The propensity-score-based weighting allowed to match the distributions of the covariates of the two groups without having to discard any data $[16,18,19]$. It was implemented based on age, relapses count (in the past 12 months), EDSS as categorized in [8], and diagnosis distance. The distributions of the covariates of each group were matched by using the population weights to estimate the average treatment effect of the population $[16,17]$, while all other settings were kept to the default settings of the twang package $[16,17]$.

To test the statistical significance of the group differences between patients receiving $\mathrm{DMT}^{*}$ and those who did not, a weighted GLM was employed according to an analysis of outcomes approach [16]. $P$-values were derived from the GLM based on the estimated significance of the relevant intercept and slope coefficients. For each observation, an indicator variable was used to specify whether the patient received $\mathrm{DMT}^{*}$. A negative binomial GLM was used for the observed number of relapses [18] and a binomial GLM was employed for the observed occurrence of a CDP as follows:

$$
\begin{gathered}
\text { svyglm.nb( observed.number.relapses } \sim \text { took.DMT } T^{*}+ \\
\text { offset } \left.\left(\log \left(\text { duration.DMT } T^{*}\right)\right), \text { design }=\text { design.ps }\right) \\
\text { svyglm }\left(\text { observed.occurence.CDP } \sim \text { took.DMT } T^{*}\right. \\
+ \text { offset }\left(\log \left(\text { duration.DMT } T^{*}\right)\right), \text { family } \\
=\text { binomial }, \text { design }=\text { design.ps })
\end{gathered}
$$

The duration of each therapy cycle was included as offset to allow for cycles with heterogeneous observation time, i.e. to allow for the comparability of the results between patients with different index therapy durations. The procedure is illustrated in Fig. 1.

\section{Results}

The following results are presented: overview of patient population after quality and inclusion criteria were applied, importance of model coefficients and model performance assessment. 


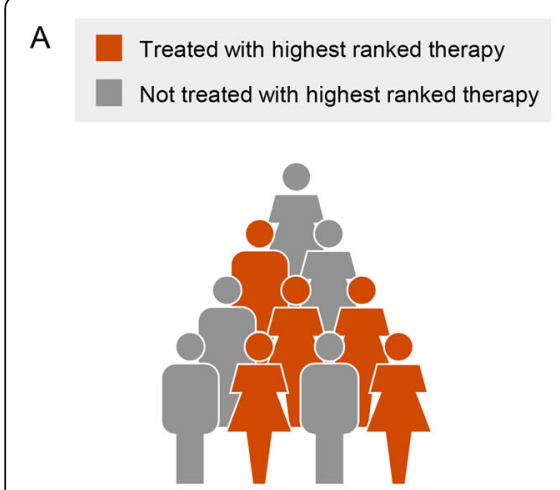

B

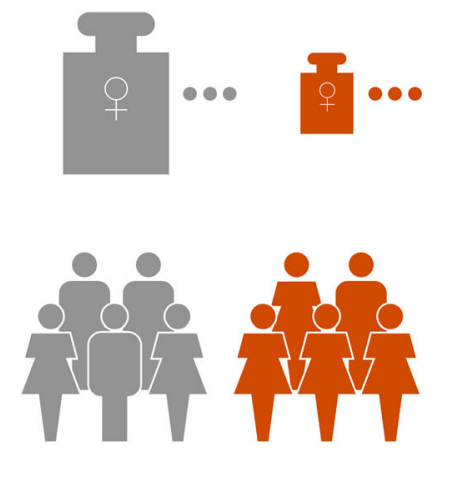

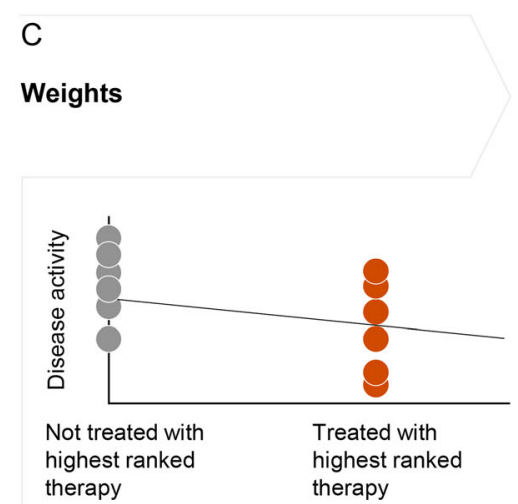

Fig. 1 Comparison of predicted therapy. a Patients assigned to the same highest ranked therapy are divided into two groups: those who received indeed this therapy (red) and those who received another therapy (gray). b Weights are calculated for the two groups to mitigate the effect of confounds on the analysis. In particular, the weights are calculated such that each group matches the population statistics. As an example, this results in larger weights for females in the group with a smaller ratio of females compared with the overall occurrence than in the group with a larger ratio. c The weights are included in a survey-weighted GLM, where an indicator variable encodes membership to one of the two groups, and observation time is accounted for. The GLM allows for a propensity-score-based weighting of the clinically relevant outcomes of the two groups. The estimated slope allows then for a comparison of the disease activity between the two groups

\section{Patient population}

After the inclusion criteria described in Section Methods: Data: Data quality and inclusion criteria have been applied, index therapy cycles consist of: Dimethylfumarat (22\%), Fingolimod (25\%), Glatirameracetat (13\%), Interferon- $\$ 1$ (19\%), Natalizumab (9\%), and Teriflunomide (13\%). At least 266 therapy cycles per DMT are available for model development. A detailed description of the patient population is reported in Additional file 1.

\section{Importance of model coefficients}

The predictors associated to the eight largest fixed-effect parameter estimates (in magnitude) of the relapse and CDP models are reported in Table 4 and Table 5, respectively. The predictors are listed along with the signs and the posteriors' median absolute deviations (MADs) of their corresponding estimates. Most of the ranked coefficients are interaction terms, two of which appear in both rankings: (i) the coefficient for the interaction between Natalizumab as index therapy and the diagnosis distance, and (ii) the coefficient for the interaction between Fingolimod as index therapy and the second-line therapy indicator. In the relapse model, the duration of the current therapy seems to have a significant impact both individually and when combined with Teriflunomide or Fingolimod. Note that the largest parameter estimate has the highest uncertainty. In the CDP model, having had a second-line DMT is particularly meaningful when the index therapy is Teriflunomide, Natalizumab or Fingolimod.

Figure 2 displays the MADs of the fixed-effects' posterior distributions in the relapse and the CDP models. In both cases, the estimates having highest uncertainty are those associated to the following predictors: duration of the current therapy, interaction between the current therapy and its duration, and Teriflunomide as a current therapy.

\section{Model performance assessment}

Model performance was assessed via model calibration, model generalizability, comparison with nested models, sensitivity analysis and comparison of observed therapy response to evaluate the usability of the models in clinical practice.

Table 4 Most important predictors in the relapse model

\begin{tabular}{lccc}
\hline Rank $^{\mathrm{a}}$ & Predictor & Sign $^{\mathrm{b}}$ & MAD \\
\hline 1 & Current $=$ TERI: Current duration & - & 4.301 \\
2 & Intercept & - & 0.553 \\
3 & Current = TERI & - & 1.406 \\
4 & Current $=$ FTY: Current duration & + & 1.983 \\
5 & Index = NA: Diagnosis distance & + & 0.368 \\
6 & Current duration & + & 1.414 \\
7 & Index $=$ TERI: Relapses count & - & 0.332 \\
8 & Index = FTY: Second-line = TRUE & - & 0.335
\end{tabular}

MAD median absolute deviation, TERI Teriflunomide, FTY Fingolimod, NA Natalizumab, Second-line DMT to be employed by label of the European Medical Agency if previous DMT failed to achieve sufficient control of disease activity.

${ }^{\text {a }}$ Ranked according to the magnitude of the median of the corresponding coefficient's posterior distribution

${ }^{b}$ A positive sign is associated with a boosting effect on the number of relapses; a negative sign is associated with a lessening effect on the number of relapses 
Table 5 Most important predictors in the CDP model

\begin{tabular}{lccc}
\hline Rank $^{\mathrm{a}}$ & Predictor & Sign $^{\mathrm{b}}$ & MAD \\
\hline 1 & Intercept & - & 0.721 \\
2 & Index = TERI: Second-line = TRUE & - & 0.977 \\
3 & Index = NA: Second-line = TRUE & - & 0.837 \\
4 & Index = NA: Diagnosis distance & + & 0.518 \\
5 & Index = FTY: Second-line = TRUE & - & 0.487 \\
6 & Current = IF: Current duration & - & 1.604 \\
7 & Current = NA: Current duration & - & 2.124 \\
8 & Current & + & 0.711 \\
\hline
\end{tabular}

MAD median absolute deviation, TERI Teriflunomide, FTY Fingolimod, NA Natalizumab, IF Interferon- $\$ 1$, Second-line DMT to be employed by label of the European Medical Agency if previous DMT failed to achieve sufficient control of disease activity.

a Ranked according to the magnitude of the median of the corresponding coefficient's posterior distribution

${ }^{\mathrm{b}}$ A positive sign is associated with a boosting effect on the likelihood of observing a CDP; a negative sign is associated with a lessening effect on the likelihood of observing a CDP

\section{Model calibration}

The agreement between predictions and observations was studied for all DMTs and also for each DMT separately.

Both the relapse model and the CDP model are well calibrated for low values of the response, while the agreement between prediction and observation worsens for high response values (Fig. 3. As only few therapy cycles have a high response value, improvement can be expected as new data is collected.

The same calibration assessment was performed for each of the six considered index therapies the models are trained on. The resulting figures show the same trend as for the calibration with all DMTs (Fig. 3), indicating that the predictions for one selected index therapy do not systematically underestimate or overestimate the response when compared with the others.

This supports the conclusion that predictions for different therapies can be compared, and that the resulting therapy ranking is usable.

\section{Model generalizability}

The generalizability of the model was assessed using three different out-of-sample validation schemes (Section Methods: Model performance assessment: Model generalizability). All results are reported in Table 6, Table 7 and Table 8.

10-fold cross-validation and test set For 10 -fold crossvalidation, the percentage change between in-sample and out-of-sample performance measures is always less than $9.5 \%$. For most performance measures and models, this change is even less than $5 \%$. The percentage change between out-of-sample and test-set performance measures is less pronounced. For the relapse model, the MSE and the negative log-likelihood drop less than $2 \%$ and the C-Index drops by $5.7 \%$. For the CDP model, the three performance measures drop by $5.7-6.3 \%$.

As the models presented in this study should be applicable to different time windows, performance measures were additionally evaluated for forecast windows between 0.5 and 5.5 years. All performance measures show the best results in the first half year, remain stable in the time window between 0.5 and 4.5 years and decline for time windows above 4.5 years. The time windows $(0.5,1.5],(1.5,2.5],(2.5,3.5],(3.5,4.5]$ show comparable results for all performance measures (Additional file 4: Table S4.1).

Leave-one-site-out cross-validation Only a slight increase in negative log-likelihood is observed when making predictions for new clinical sites compared with in-sample predictions. Furthermore, the negative log-likelihood has a similar variability within and across clinical sites for both the relapse and CDP models (Additional file 9). These findings indicate that the models generalize well to new clinical sites.

In summary, the results presented in this section indicate that the relapse model is more robust than the CDP model and that both predictive models are robust and therefore able to generalize to new patients and clinical sites, especially when predicting up to 4.5 years into the future.

\section{Comparison with nested models}

Two additional nested models of lower complexity were presented in Section Methods: Model performance assessment: Comparison with nested models. Predictive models were compared with these non-personalized and prognostic models based on out-of-sample performance measures (40 times 10-fold cross-validation).

\section{Comparison between predictive and non-personalized} model When adding personal characteristics to the relapse model, an increase of performance is observed in both discrimination (increase of C-Index from 0.5531 to 0.6458 by 0.0927 ) and goodness-of-fit (decrease of negative log-likelihood of -69.3), while no change is observed for the MSE (-0.0003). For the CDP model, the results derived from the performance measures are inconclusive. The negative log-likelihood and the MSE show a decrease in performance from the non-personalized to the predictive model (5.8 resp. 0.00048 difference). On the other hand, the addition of the personal characteristics causes an improvement of the C-Index by 0.0352 .

Comparison between predictive and prognostic model The predictive models include additional parameters accounting for interactions between patient characteristics and index therapy compared with the prognostic model (Table 3), which allows the predicted therapy effect to differ for different patients (Section Methods: Model 


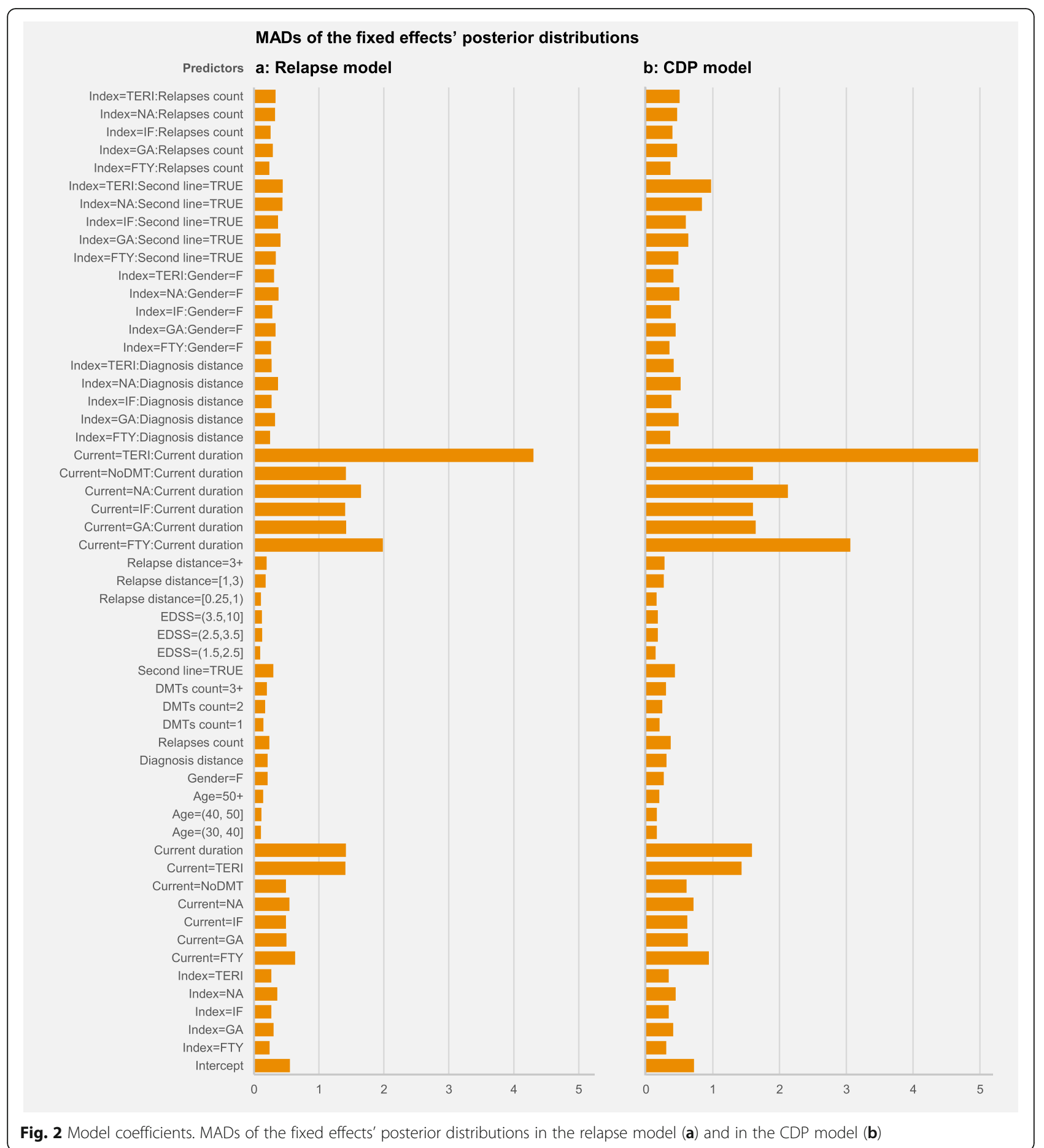

performance assessment: Comparison with nested models). For the relapse model, a decrease in performance is observed when accounting for interactions (CIndex - 0.0024, MSE 0.0242, negative log-likelihood 6.2). For the CDP model, a decrease is observed in goodnessof-fit (MSE 0.00011, negative log-likelihood - 1.4) while an increase in performance is observed for the C-Index (0.0194).
In summary, adding personal characteristics as predictors is beneficial in terms of C-Index and negative loglikelihood, for the relapse model, and in terms of C-Index, for the CDP model. These findings suggest that this first level of personalization is worth to be further investigated. Adding a second level of personalization, i.e. interactions between patient characteristics and index therapy, increases model complexity and shows no improvement in 
Mean observed number of relapses per prediction bin ( $y$ axis) vs. Mean predicted number of relapses (x axis) for n-plet DMF, FTY, GA, IF, NA, TERI

a

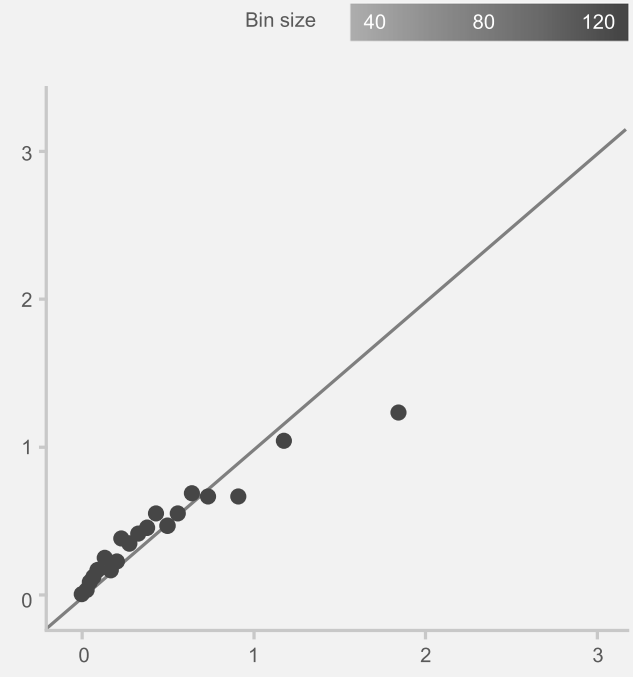

b
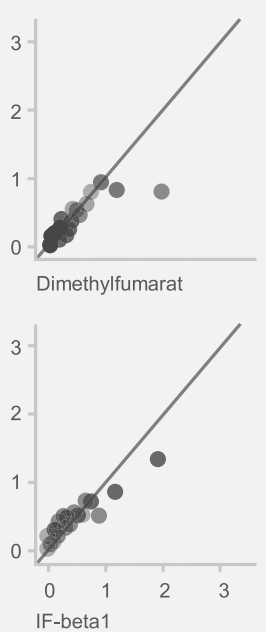

Bin size

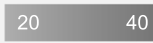

80

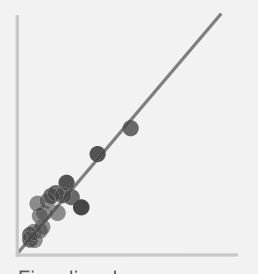

Fingolimod
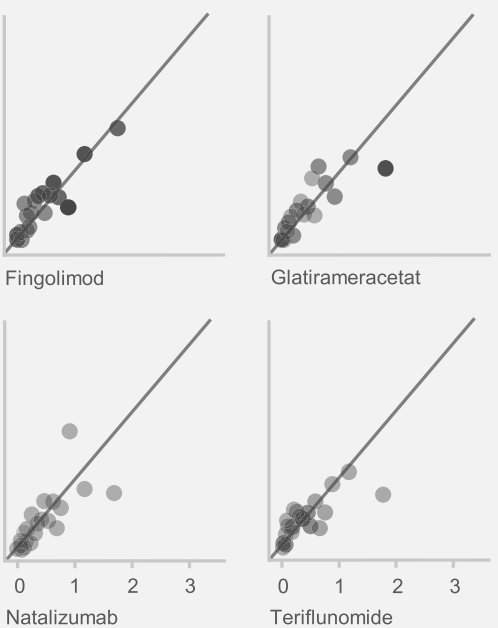

Glatirameracetat

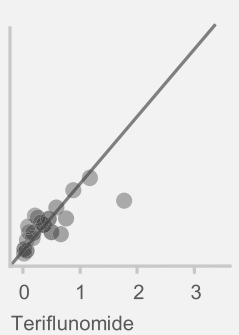

Mean observed proportion of CDP per prediction bin (y axis) vs. Mean predicted probability of CDP (x axis) for n-plet DMF, FTY, GA, IF, NA, TERI

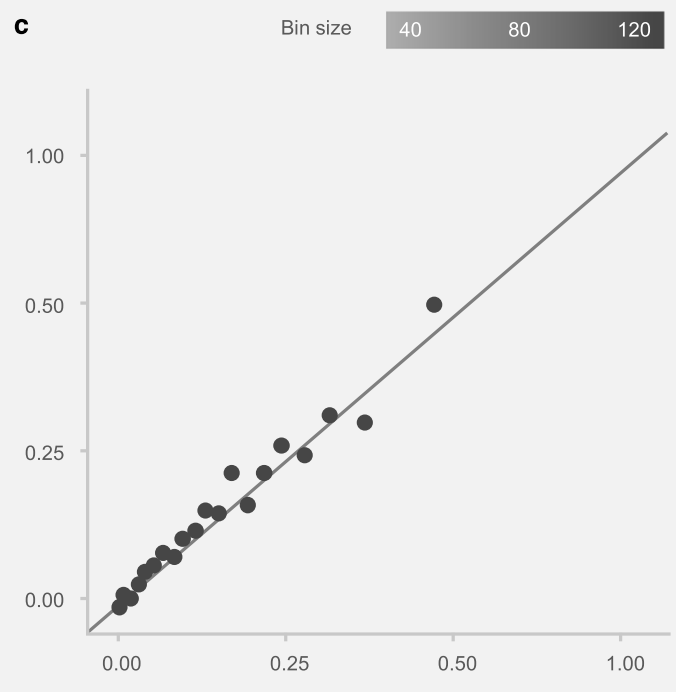

d
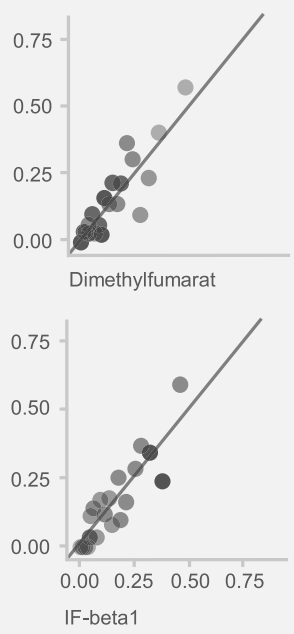

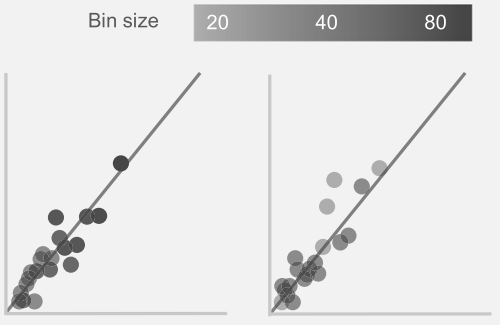

Fingolimod

Glatirameracetat
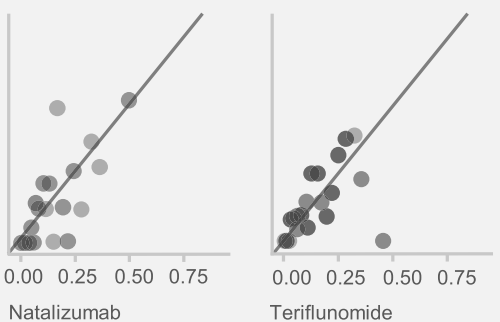

Fig. 3 Calibration. Calibration of the relapse model (top) and CDP model (bottom) using equally-populated bins, when considering all DMTs together (left) and when considering each DMT separately (right). The predictions are split into 20 equally-populated bins from zero to four relapses, for the relapse model, and from zero to one, for the CDP model. Different shades of gray highlight a different population size

the performance measures for the relapse model, and an improvement in the C-Index and negative log-likelihood of the CDP model. In order to further assess the benefit of the predictive models, a new method was introduced in Section Methods: Model performance assessment: Comparison of predicted therapy response and is evaluated below in Section Results: Model performance assessment: Comparison of predicted therapy response. This allows to investigate the potential of these models from a different perspective.

\section{Model robustness}

It was shown that the predictive models are robust against different choices of the priors, as for the vast majority of patients the changes in the model coefficients did not affect the therapy ranking (Additional file 5: Figure S5.7). 
Table 6 Performance of predictive, prognostic and non-personalized models based on out-of-sample and in-sample predictions

\begin{tabular}{|c|c|c|c|c|c|}
\hline Measure & Out-of-sample mean (SE) ${ }^{a}$ & In-sample mean (SE) ${ }^{a}$ & Sample size ${ }^{b}$ & Response & Model \\
\hline C-Index & $0.5819(0.0008)$ & $0.6546(0.0005)$ & 307,784 & CDP & predictive \\
\hline C-Index & $0.5625(0.0007)$ & $0.6220(0.0004)$ & 307,784 & CDP & prognostic \\
\hline C-Index & $0.5467(0.0006)$ & $0.5649(0.0005)$ & 307,784 & CDP & non-personalized \\
\hline C-Index & $0.6458(0.0004)$ & $0.6781(0.0003)$ & 505,724 & relapse & predictive \\
\hline C-Index & $0.6482(0.0003)$ & $0.6700(0.0002)$ & 505,724 & relapse & prognostic \\
\hline C-Index & $0.5531(0.0003)$ & $0.5609(0.0003)$ & 505,724 & relapse & non-personalized \\
\hline MSE & $0.12497(0.00005)$ & $0.11928(0.00004)$ & 3119 & CDP & predictive \\
\hline MSE & $0.12486(0.00004)$ & $0.12132(0.00003)$ & 3119 & CDP & prognostic \\
\hline MSE & $0.12449(0.00002)$ & $0.12388(0.00001)$ & 3119 & CDP & non-personalized \\
\hline MSE & $0.7554(0.0008)$ & $0.7097(0.0006)$ & 3119 & relapse & predictive \\
\hline MSE & $0.7312(0.0005)$ & $0.7049(0.0003)$ & 3119 & relapse & prognostic \\
\hline MSE & $0.7557(0.0002)$ & $0.7517(0.0001)$ & 3119 & relapse & non-personalized \\
\hline NLL & $1252.6(0.6)$ & $1190.9(0.4)$ & 3119 & CDP & predictive \\
\hline NLL & $1254.0(0.5)$ & $1215.5(0.2)$ & 3119 & CDP & prognostic \\
\hline NLL & $1246.8(0.2)$ & $1240.3(0.1)$ & 3119 & CDP & non-personalized \\
\hline NLL & $2580.8(0.6)$ & $2519.9(0.4)$ & 3119 & relapse & predictive \\
\hline NLL & $2574.6(0.5)$ & $2534.9(0.3)$ & 3119 & relapse & prognostic \\
\hline NLL & $2650.1(0.2)$ & $2641.9(0.2)$ & 3119 & relapse & non-personalized \\
\hline
\end{tabular}

SE standard error of the mean, CDP confirmed disability progression, C-Index Harrell's concordance statistic, MSE mean squared error, NLL negative log-likelihood.

${ }^{a}$ Estimated by repeating 10 -fold cross-validation 40 times

${ }^{b}$ Refers either to the number of observations (MSE, NLL) or the number of matched pairs (C-Index)

Exceptions were cases where the predictions for different therapies were already similar. Similarly, the predictive models proved robust against differing characteristics of the underlying patient population and against changes in the sample size within the assessed range (Additional file 6 and Additional file 7).

\section{Comparison of predicted therapy response}

Both predictive models enable to derive a personalized ranking of therapies for each patient (Section Methods: Model performance assessment: Comparison of predicted therapy response). In the following, disease activity is compared within a survey-weighted GLM between

Table 7 Performance of models trained on the full data set and evaluated on the test set

\begin{tabular}{lcccc}
\hline Measure & Value & Sample size $^{\text {a }}$ & Response & Model \\
\hline C-Index & 0.554 & 3354 & CDP & predictive \\
C-Index & 0.608 & 5606 & relapse & predictive \\
Average NLL & 0.423 & 314 & CDP & predictive \\
Average NLL & 0.821 & 314 & relapse & predictive \\
MSE & 0.131 & 314 & CDP & predictive \\
MSE & 0.784 & 314 & relapse & predictive
\end{tabular}

CDP confirmed disability progression, C-Index Harrell's concordance statistic, MSE mean squared error, NLL negative log-likelihood.

a Refers either to the number of observations (MSE, average NLL) or the number of matched pairs (C-Index) patients being treated with the highest ranked therapy $\mathrm{DMT}^{*}$ and patients treated with a different DMT.

From the six therapies discussed in this study (Dimethylfumarat, Fingolimod, Glatirameracetat, Interferon- $ß 1$, Natalizumab, and Teriflunomide) only those which had both patients receiving and not receiving DMT* could be considered. Furthermore, implementation properties of the employed packages required a minimum of 10 observations per condition [20]. Due to these criteria, Glatirameracetat, Interferon- $\beta 1$ and Teriflunomide had to be excluded from the assessment of the relapse model and Interferon- $\beta 1$ had to be excluded from the assessment of the CDP model (Additional file 8: Table S8.1).

The results of the relapse model are presented in Table 9. This table summarizes the group comparisons derived from the survey-weighted negative binomial GLM svyglm.nb (Section Methods: Model performance assessment: Comparison of predicted therapy response), applied to the observed number of relapses and for the respective DMT*. Observation time has been accounted for within this GLM. As the indicator variable takes value 1 for receivers of DMT* and 0 for non-receivers of DMT*, a negative slope indicates a lower disease activity for patients who received DMT*. Both Dimethylfumarat and Natalizumab are associated with a significantly lower number of observed relapses at the $p<0.05$ significance level when patients received DMT* compared to other DMTs. 
Table 8 Performance of the predictive models based on leave-one-site-out cross-validation

\begin{tabular}{|c|c|c|c|c|c|}
\hline Measure & Out-of-sample ${ }^{a}$ mean & In-sample ${ }^{b}$ mean & Sample size ${ }^{c}$ & Response & Model \\
\hline C-Index & 0.579 & 0.652 & 307,784 & CDP & predictive \\
\hline C-Index & 0.646 & 0.675 & 505,724 & Relapse & predictive \\
\hline MSE & 0.125 & 0.119 & 3119 & CDP & predictive \\
\hline MSE & 0.748 & 0.711 & 3119 & Relapse & predictive \\
\hline NLL & 1254.330 & 1192.544 & 3119 & CDP & predictive \\
\hline NLL & 2581.261 & 2523.731 & 3119 & Relapse & predictive \\
\hline
\end{tabular}

CDP confirmed disability progression, C-Index Harrell's concordance statistic, MSE mean squared error, NLL negative log-likelihood.

${ }^{a}$ Based on predictions for patients from a clinical site not used during training

${ }^{\mathrm{b}}$ Based on predictions for all patients from all clinical sites used for training

${ }^{c}$ Refers either to the number of observations (MSE, NLL) or the number of matched pairs (C-Index)

Fingolimod, however, is associated with lower sample sizes in both groups, and the differences between the two groups are not significant.

The results of the CDP model are presented in Table 10. This table summarizes the group comparisons derived from the survey-weighted quasi-binomial GLM svyglm (Section Methods: Model performance assessment: Comparison of predicted therapy response), applied to the observed occurrences of $\mathrm{CDP}$, and for the respective DMT*. Analogously to the assessment of the relapse model, observation time has been accounted for within this GLM and a negative slope indicates a lower disease activity for patients who received DMT*. All therapies except Fingolimod are associated with a lower occurrence of CDP when patients received DMT*, although only the differences for Dimethylfumarat are statistically significant at the significance level of $p<0.05$. The remaining therapies are associated with considerably smaller sample sizes in at least one of the two groups.

In summary, recommendations based on predicted rankings could not be assessed for all six therapies due to sample size (Additional file 8: Table S8.1). For the CDP model, five of the six therapies could be assessed, where a negative estimated slope from a surveyweighted GLM corresponds to the highest ranked therapy in four cases, and is significant for one comparison. For the relapse model, three of the six therapies were assessed, where a negative estimated slope from a survey-weighted GLM is associated with the highest ranked therapy in all cases except for Fingolimod, and is significant for two comparisons. These results indicate that although performance measures do not capture the additional benefit of interactions between patient characteristics and index therapy, the comparison of treatment effectiveness derived from both predictive models adds value in clinical practice, as patients receiving $\mathrm{DMT}^{*}$ show less disease activity than patients receiving any other DMT (significant for three out of eight comparisons).

\section{Discussion}

A framework for personalized prediction was employed to assess the effectiveness of different DMTs with regards to clinical outcomes of RRMS. Hierarchical Bayesian GLMs were implemented to predict the number of relapses or the occurrence of a CDP, for several available DMTs. The predictive framework was based on real-world data collected in the NTD registry, which consists of clinical data on patient characteristics and disease history.

Assessment of the model performance using established statistical methods demonstrated that the relapse model and the CDP model provide robust and accurate predictions, and that both models generalize to new patients and clinical sites.

The predictive relapse model achieved an average outof-sample C-Index of 0.65 and an average out-of-sample MSE of 0.76 relapses. The predictive CDP model achieved an average C-Index of 0.58 and an average outof-sample MSE of 0.12 CDPs.

The predictive models were shown to be robust against different choices of the priors and against sample size. Robustness against different choices of the priors was proven by the fact that changing the prior distributions does not influence the predicted therapy ranking. Exceptions were cases where the predictions for different

Table 9 Comparison of therapy effectiveness for the relapse model

\begin{tabular}{|c|c|c|c|c|}
\hline$\overline{\mathrm{DMT}^{*}}$ & Slope coefficient ${ }^{\mathrm{a}}$ & Sample size when DMT* was taken & Sample size when DMT* was not taken & $p$-value \\
\hline Dimethylfumarat & -0.6918 & 134 & 307 & 0.016 \\
\hline Fingolimod & 0.0423 & 128 & 112 & 0.860 \\
\hline Natalizumab & -0.4376 & 234 & 2182 & 0.019 \\
\hline
\end{tabular}

$D M T^{*}$ highest ranked disease modifying therapy.

${ }^{a}$ Derived from a survey-weighted negative binomial generalized linear model where negative sign indicates lower disease activity 
Table 10 Comparison of therapy effectiveness for the CDP model

\begin{tabular}{|c|c|c|c|c|}
\hline $\mathrm{DMT}^{*}$ & Slope coefficient ${ }^{a}$ & Sample size when DMT* was taken & Sample size when DMT* was not taken & $p$-Value \\
\hline Dimethylfumarat & -0.5363 & 863 & 306 & 0.027 \\
\hline Fingolimod & 0.1114 & 135 & 87 & 0.792 \\
\hline Glatirameracetat & -0.5336 & 238 & 45 & 0.350 \\
\hline Natalizumab & -0.4021 & 1101 & 132 & 0.405 \\
\hline Teriflunomide & -0.4317 & 179 & 16 & 0.730 \\
\hline
\end{tabular}

$D M T^{*}$ highest ranked disease modifying therapy.

${ }^{a}$ Derived from a survey-weighted quasi-binomial generalized linear model where negative sign indicates lower disease activity

therapies were already similar. Robustness against sample size was proven by the fact that model coefficients do not change significantly when reducing the sample size within the assessed range.

The overall performance of the predictive models allows for reliable comparisons of the effectiveness of different therapies. Therapy rankings obtained from both predictive models proved to be aligned with real-world clinical outcomes. Patients having received the therapy with the highest predicted effectiveness were less likely to suffer from relapses or to experience a CDP compared with patients treated with other DMTs. Statistically significant differences were always associated with better disease outcomes for patients who received the highest ranked therapy.

The generalizability of the models was evaluated by implementing a 10-fold cross-validation scheme, a leave-one-site-out cross-validation scheme with respect to the clinical site, and by using a test set. When comparing in-sample against out-of-sample performance measures, performance never dropped by more than $9.5 \%$ for all three performance measures used in this study, indicating that the predictive models generalize well to an unseen patient population. Comparable performance was obtained using 10fold cross-validation and the test set. This indicates that the predictive models can be applied to new patients that fulfill the inclusion criteria. The availability of a large multi-site dataset with high-quality entries in the NTD registry proved important for testing the generalizability of the models to new clinical sites, an inevitable requirement to ensure applicability to a wide range of patients.

As application of predictive models in RRMS is still at an early stage, this study builds on previous studies in this field $([8,9,21,22])$ and addresses identified challenges: (1) effectiveness of different therapies with regards to disease course can be compared with one another as they are based on the same model and underlying data, (2) the models fulfill multiple established statistical criteria for accuracy and robustness, and show comparable performance for predictions up to 4.5 years into the future, (3) the models generalize to new patients and new clinical sites, (4) model predictions are clinically meaningful, since patients receiving the therapy with the highest predicted effectiveness had a better observed clinical outcome in terms of number of relapses and CDP.

The Bayesian predictive modeling setup is ideal for including newly available therapies, as results derived from clinical studies can be used as prior information. Predictive models will be updated on a regular basis every three months, following the continuous updates of the NTD registry. For this, the predictive models are fitted based on an updated training set after quality and inclusion criteria have been applied to a new data extraction, and the main validation routine as described above is performed. After quality checks have been passed, coefficients are updated accordingly. This will ensure that the underlying algorithms are monitored and validated regularly, and that changes in the RRMS treatment landscape will be captured as soon as possible. In addition, new models will be assessed to improve the capabilities of the framework even further.

For the presented and all further extensions of the predictive models, additional validation on external data sets is planned. As magnetic resonance imaging (MRI) and molecular data for the prediction of RRMS treatment response become available, their usefulness will be evaluated to improve predictive accuracy and tailor the predictive framework even further to personalized information.

\section{Conclusion}

The findings presented in this study support a personalized approach in RRMS treatment and tackle some caveats in classical medicine, where assessing the potential individual benefit of therapy choices is based on grouplevel analysis and not on individual patient characteristics [23] [24] [25] [26]. Predictive models that are continuously updated and that provide personalized comparative therapy effectiveness insight support the 
multifaceted shared decision process between doctors and their patients in a clinically meaningful way. Positive experience of their use in daily clinical practice will foster collection of high-quality real-world data and contribute to the transition to effective personalized treatment of RRMS.

\section{Supplementary information}

Supplementary information accompanies this paper at https://doi.org/10. 1186/s12874-020-0906-6.

Additional file 1. "Inclusion criteria and resulting patient population":

Supplementary tables and results.

Additional file 2. "CDP definition": Definition of confirmed disability progression.

Additional file 3. "Mathematical model description": Supplementary information to Section Methods: Model development.

Additional file 4. "Performance by observation time window": Supplementary table.

Additional file 5. "Model robustness against different prior choices": Supplementary methods and results.

Additional file 6. "Model robustness against differences in patient characteristics": Supplementary methods and results.

Additional file 7. "Model robustness against different training set size": Supplementary methods and results.

Additional file 8. "Comparison of predicted treatment effectiveness": Supplementary tables.

Additional file 9. "Clinical site effect": Supplementary figures.

\section{Abbreviations}

CDP: Confirmed disability progression; DMF: Dimethylfumarat; DMT: Diseasemodifying therapy; EDSS: Expanded Disability Status Scale; FTY: Fingolimod; GA: Glatirameracetat; GLM: Generalized linear model; IBE: Institut für medizinische Informationsverarbeitung, Biometrie und Epidemiologie; IF: Interferon-B1; MAD: Median absolute deviation; MRI: Magnetic resonance imaging; MS: Multiple sclerosis; MSE: Mean squared error; NA: Natalizumab; NLL: Negative log-likelihood; NTD: NeuroTransData; RCT: Randomized clinical trial; RRMS: Relapsing-remitting multiple sclerosis; SE: Standard error; TERI: Teriflunomide

\section{Acknowledgements}

We thank Knut Wehrle and Fabian Roßnagel (both NeuroTransData) for their technical and organizational support and all members of NeuroTransData for their dedication and enthusiasm to collect clinical data and to continuously improve data quality for more than 10 years. We thank Prof. Lu Tian and Prof. Torsten Hothorn for valuable feedback to the manuscript. Furthermore, we would like to thank Fabienne Hasler, Maggie Tischhauser, Sandra Müller and Severin Eichenberger (PwC document production center, Switzerland) for the realization of the figures. We thank Dr. Fabio Pellegrini and Dr. Marin Puskaric for valuable feedback and Linda Vos for her support throughout the project.

\section{Authors' contributions}

Lead and planning: $\mathrm{P} \vee \mathrm{H}, \mathrm{AB}$. Writing - original draft, review and editing: $\mathrm{ES}$, $\mathrm{SB}, \mathrm{FL}, \mathrm{YH}, \mathrm{PKF}, \mathrm{EJ}, \mathrm{CW}$. Original Conceptualization: ES. Statistical Analysis and Implementation: ES, FL, YH, PKF, EJ. Review of Statistical Analysis: PvH, SB, AB. Data curation: SB, AB. All authors read and approved the final manuscript.

\section{Funding}

Not applicable.

\section{Availability of data and materials}

The datasets generated and analyzed during the current study are not publicly available due to them containing information that could compromise research participant privacy but are available from the corresponding author SB on reasonable request.

\section{Ethics approval and consent to participate}

All clinical data captured are pseudonymized and pooled to form the NeuroTransData MS registry database. The codes uniquely identifying patients are managed by the Institute for Medical information processing, Biometry and Epidemiology (Institut für medizinische Informationsverarbeitung, Biometrie und Epidemiologie (IBE)) at the Ludwig Maximilian University in Munich, Germany, acting as an external trust center. The data acquisition protocol described in Section Methods: Data was approved by the ethical committee of the Bavarian Medical Board (Bayerische Landesärztekammer; June 14, 2012) and re-approved by the ethical committee of the Medical Board North-Rhine (Ärztekammer Nordrhein, 25.04.2017).

\section{Consent for publication}

Not applicable.

\section{Competing interests}

The authors declare that they have no competing interests.

\section{Author details}

${ }^{1}$ PwC Digital Services, Zürich, Switzerland. ${ }^{2}$ NeuroTransData, Neuburg an der Donau, Germany.

Received: 31 January 2019 Accepted: 20 January 2020

\section{2.}

\section{References}

1. Grossman I, Knappertz V, Laifenfeld D, Ross C, Zeskind B, Kolitz S, et al. Pharmacogenomics strategies to optimize treatments for multiple sclerosis: insights from clinical research. Neurobiology. 2017;152:114-30.

2. EMA Human Scientific Committees' Working Parties with Patients' and Consumers' Organisations (PCWP) and Healthcare Professionals' Organisations (HCPWP). Regulatory perspective on real world evidence (RWE) in scientific advice. https://www.ema.europa.eu/documents/ presentation/presentation-regulatory-perspective-real-world-evidence-rwescientific-advice-emas-pcwp-hcpwp-joint_en.pdf. Accessed 25112018.

3. Eichler HG, Bloechl-Daum B, Broich K, Kyrle PA, Oderkirk J, Rasi G, et al. Data rich, information poor: Can we use electronic health records to create a learning healthcare system for pharmaceuticals? Clin Pharmacol Ther. 2018;0(0).

4. Dreyer NA. Advancing a framework for regulatory use of real-world evidence: when real is reliable. Ther Innov Regul Sci. 2018;52(3):362-8.

5. STAMP Commission Expert Group.. https://ec.europa.eu/health/sites/health/ files/files/committee/stamp/stamp_9_41_2_en.pdf. Accessed 25112018.

6. Sherman RE, Anderson SA, Dal Pan GJ, Gray GW, Gross T, Hunter NL, et al. Real-world evidence - what is it and what can it tell us? N Engl J Med. 2016;375:2293-7.

7. D'Amico E, Patti F, Zanghi A, Zappia M. A personalized approach in progressive multiple sclerosis: The current status of disease modifying therapies (DMTs) and future perspectives. Int J Mol Sci. 2016;17(10).

8. Engler D, Tanuja C, Healy B. Joint assessment of dependent discrete disease state processes. Stat Methods Med Res. 2015.

9. Stangel M, Penner IK, Kallmann BA, Lukas C, Kieseier BC. Towards the implementation of 'no evidence of disease activity' in multiple sclerosis treatment: the multiple sclerosis decision model. Ther Adv Neurol Disord. 2015:8(1):3-13.

10. Gelman A, Rubin DB. Inference from iterative simulation using multiple sequences. Stat Sci. 1992;7(4):457-72.

11. Frank E, Harrell J, Kerry L, Daniel B. Tutorial in biostatistics multivariable prognostic models: issues in developing models, evaluating assumptions and adequacy, and measuring and reducing errors. Stat Med. 1996;15:361-87.

12. Gelman A, Stern HS, Carlin JB, Dunson DB, Vehtari A, Rubin DB. Bayesian data analysis: Chapman and Hall/CRC; 2013.

13. Stan Development Team. RStan: The R interface to Stan. Version 2.16.0. 2017.

14. Prior Choice Recommentations. 2018. https://github.com/stan-dev/stan/ wiki/Prior-Choice-Recommendations. Accessed 30012019

15. Steyerberg EW, Vickers AJ, Cook NR, Gerds T, Gonen M, Obuchowski N, et al. Assessing the performance of prediction models: a framework for some traditional and novel measures. Epidemiology. 2010;21(1):128.

16. Ridgeway G, McCaffrey D, Morral A, Burgette L, Griffin BA. Toolkit for weighting and analysis of nonequivalent groups: a tutorial for the twang package; 2017. 
17. Ridgeway G, McCaffrey D, Morral A, Griffin BA, Burgette L. Twang: Toolkit for Weighting and Analysis of Nonequivalent Groups https://cran.r-project.org/ web/packages/twang/index.html. Accessed October 2018.

18. Rosenbaum PR, Rubin DB. The central role of the propensity score in observational studies for causal effects. Biometrika. 1983;70(1):41-55.

19. Lüdecke D. Sjstats: Statistical functions for regression models (version 0.17.1). 2018. https://CRAN.R-project.org/package=sjstats.

20. Ridgeway G, et al. gbm: Generalized boosted regression models. 2017 [R package version 2.1.3]. https://CRAN.R-project.org/package $=g b m$.

21. Harrell FE Jr, Frank E. Regression modeling strategies: with applications to linear models, logistic and ordinal regression, and survival analysis: Springer; 2015.

22. Ikeda M, Ishigaki T, Yamauchi K. Relationship between brier score and area under the binormal ROC curve. Comput Methods Prog Biomed. 2002;67(3):187-94.

23. Montalban X, Gold R, Thompson AJ, Otero-Romero S, Amato MP, Chandraratna $D$, et al. ECTRIMS/EAN guideline on the pharmacological treatment of people with multiple sclerosis. Mult Scler J. 2018;24(2):96-120.

24. Deutsche Gesellschaft für Neurologie. Leitlinie Multiple Sklerose. https:// www.awmf.org/leitlinien/detail/l/030-050.html. Accessed 22 January 2019.

25. Rae-Grant A, Day GS, Marrie RA, Rabinstein A, Cree BA, Gronseth GS, et al. Practice guideline recommendations summary: disease-modifying therapies for adults with multiple sclerosis: Report of the Guideline Development, Dissemination, and Implementation Subcommittee of the American Academy of Neurology. Neurology. 2018;90(17).

26. National Institute for Health and Care Excellence. Multiple sclerosis in adults: management. 2014. https://www.nice.org.uk/guidance/cg186. Accessed 22 Jan 2018.

\section{Publisher's Note}

Springer Nature remains neutral with regard to jurisdictional claims in published maps and institutional affiliations.

Ready to submit your research? Choose BMC and benefit from:

- fast, convenient online submission

- thorough peer review by experienced researchers in your field

- rapid publication on acceptance

- support for research data, including large and complex data types

- gold Open Access which fosters wider collaboration and increased citations

- maximum visibility for your research: over $100 \mathrm{M}$ website views per year

At $\mathrm{BMC}$, research is always in progress.

Learn more biomedcentral.com/submissions 